ROCZNIKI TEOLOGICZNE

Tom LXVIII, zeszyt $7-2021$

DOI: https://doi.org/10.18290/rt21687-2

PÉTER SZENTPÉTERY

\title{
THEOLOGIE, RELIGIONSWISSENSCHAFT UND THEOLOGISCHER UNTERRICHT. EINIGE IMMER NEU ZU ENTDECKENDE GEDANKENSPLITTER
}

\author{
THEOLOGY, RELIGIOUS STUDIES AND THEOLOGICAL EDUCATION. \\ SOME SHARDS OF THOUGHT TO ALWAYS BE DISCOVERED ANEW
}

\begin{abstract}
A b stract. The author collected a few insights which he would consider as important concerning religiosity and religions in theological education. According to the first part it should be clear for future pastors, church workers and teachers of religion that they are always challenged by the question of the origin of religiosity, humanity and morality. The key question lying behind these is: What is the human being as such? The second part deals with the question of what objectivity in religious studies means and how much it is different in theology. It cannot be identified with agnosticism or even atheism. Any student of theology has to study other religions in their context, the history of their origins with utmost understanding and empathy which need not necessarily mean agreement. This leads to the third part on interreligious and ecumenical dialogue. Personal contacts cannot be substituted even with the best upto-date literature. Dialogue does not mean self-abandonment, because that would make it unserious. Principles in ecumenical, i.e. inter-church dialogue are basically the same, although it is much more what unites than divides the partners. Briefly: we are truth-seekers. Finally a conclusion is summed up in nine theses.
\end{abstract}

Keywords: theology; religious studies; religiosity; origin of religion; dialogue; morality; objectivity; tolerance.

Dr. PÉTER SZENTPÉTERY - Privatdozent an der Evangelisch-Lutherischen Theologischen Universität (Kirchliche Hochschule) in Budapest. Er unterrichtet Konfessionskunde/Ökumene, Religionsgeschichte, Weltreligionen und beschäftigt sich mit Fragen von Glauben und Wissen, in seinem Habilitationsbuch mit Darwins Wirkung auf den Schöpfungsglauben (2008). Er war zwischen 2000-2015 Sekretär des Ökumenischen Studienzentrums, Budapest, und 2012-2014 Sekretär der Societas Oecumenica; Korrespondenzadresse: e-mail: peter.szentpetery@lutheran.hu 
Ich glaube, daß mich Gott geschaffen hat samt allen Kreaturen. (Martin Luther) ${ }^{1}$

Die folgenden Gedanken wollen nicht den Anspruch auf Neuigkeit und Erfindung erheben, es kann wohl sein, dass einige, sogar die meisten von ihnen für viele als überholt erscheinen. Doch denke ich, im theologischen Unterricht muss man sie überlegen und mit den künftigen Pfarrern, Religionslehrern, Katecheten und Gemeindemitarbeitern teilen um ihnen die Besonderheit und Unentbehrlichkeit ihres Berufs und Berufung bewusst zu machen. Dietrich Bonhoeffer sowie das Projekt Weltethos und die neuen Atheisten sind uns schon dazu zur Hilfe gekommen. ${ }^{2}$

\section{RELIGION, RELIGIONSWISSENSCHAFT UND URSPRUNG}

Jede Erklärung des Ursprungs der Religion setzt schon eine religiösweltanschauliche Grundhaltung voraus. In allen rationalisierenden Erklärungsversuchen - von der Personifizierung von Naturgewalten bzw. menschlichen Eigenschaften bis zum „Opium für das Volk“ - ist mehr oder weniger Wahrheit enthalten. Da es um ein einmaliges Ereignis oder um einen (wie auch immer langen) Prozess geht, können keine dieser - sonst auf wiederholbaren Beobachtungen basierende - Versuche eine vollständige Erklärung liefern, auch ihr Zusammenschluss nicht. Sie können nur gewisse Phänomene - manchmal sehr gut, überzeugend - erklären, aber nichts mehr. Nehmen wir z. B. Feuerbachs Erklärung mit der Personifizierung von Naturgewalten und menschlichen Eigenschaften. Niemand kann leugnen, dass es oftmals wirklich darum geht. Woher kommt aber der Anspruch bzw. die Fähigkeit des Menschen, diesen Weg zu begehen? Oder illustriert gerade nicht die zur Reformation führende Geschichte mit den Ablassbriefen, dass Religion wirklich Opium für das Volk sein kann? Wer konnte aber den/die ersten religiösen Menschen, oder - wenn man aus der Untrennbarkeit von Mensch und Religion ausgeht, den/die ersten - religiösen - Menschen beobachten?

\footnotetext{
${ }^{1}$ Martin Luther, „Der Kleine Katechismus,“ w Unser Glaube. Die Bekenntnissschriften der evangelisch-lutherischen Kirche. Ausgabe für die Gemeinde, Bearbeitet von Host Georg Pöhlmann (Gütersloh: Gütersloher Verlagshaus Gerd Mohn, 1986), 542 f.

${ }^{2}$ Péter Szentpétery, „Dietrich Bonhoeffer und der heutige religiöse Pluralismus,“ Roczniki Teologiczne 62(2015), 7: 121-135. Ders.: „Weltethos, neuer Atheismus und christliche Identität,“ Roczniki Teologiczne 66(2019), 7: 69-86.
} 
Religion strebt sich nach dem letztendlichen Verständnis der Dinge, sie ist also keine bloße Frage bzw. kein Ausdrucksmittel des Gefühls, der Angst, der Vorstellungskraft oder der Stimmung. Man könnte hier Viktor Frankl, den Vater der Logotherapie nennen -, der Mensch sucht irgendeinen Sinn für sein Leben (also Logos im Sinne der Sinnfrage). „Ob er es will, oder nicht, ob er es wahrhat oder nicht - der Mensch glaubt an einen Sinn, solange er atmet. “3 Füge man noch mit Veli-Matti Kärkkäinen hinzu: Das Leben des Menschen hat nur Sinn, wenn dieser gewaltige Kosmos Sinn hat, so wie im Falle eines Autos. Steuerrad, Bremse usw. haben Sinn nur im Zusammenhang eines funktionsfähigen Fahrzeuges. ${ }^{4}$ Sagen wir so nachWolfhart Pannenberg: der Mensch ist das einzige auf die Welt, auf die Unendlichkeit hin offene Wesen, er hat also eine spezielle, ausgezeichnete Lage in der Welt. ${ }^{5}$ Diese spezielle Lage muss auch zu ihrer Ablehnung vorausgesetzt werden. Man kann hier z. B. dem Begriff des Unendlichen nachdenken. Kann der Mensch wirklich nur als Produkt, sein Geist als Epiphänomen bloßer physikalischer Prozesse aufgefasst werden? Alle physikalischen Prozesse sind per definitionem endlich. Die Möglichkeit zum Denken an das Unendliche ist aber unendlich. Der Begriff des Endlichen hat Sinn nur in Gegenüberstellung mit dem Unendlichen. ${ }^{6}$ Ontologischer Reduktionismus ist eine Sackgasse, ein Widerspruch: der Mensch ist nichts mehr, nichts anders als ... ${ }^{7}$ Der Mensch muss

${ }^{3}$ Viktor E. Frankl, Der Mensch auf der Suche nach Sinn. Zur Rehumanisierung der Psychotherapie (Freiburg-Basel-Wien: Herder Verlag, ${ }^{4}$ 1975), 116. Er meint, „für die Logotherapie kann Religion kein Standort, nur Gegenstand sein“ (113.), doch liegt sie ihr ,zumindest sehr am Herzen“ (115.)

${ }^{4}$ Veli-Matti Kärkkäinen, „Meaningful life in a meaningless cosmos? A short reflection for good times - and bad,“ Dialog. Journal of Theology 59(2020): 103-106. „Let me give you a childlike example: while the steering wheel or the brakes in the car may serve a particular purpose in the car, it does not make sense to speak of their meaningfulness unless the car itself is meaningful; without the rest of the car, it doesn't matter if they function properly or not!“ (104.) Man kann die (rhetorischen) Fragen von Hans-Dieter Mutschler gegenüberstellen: „... dieser gewaltige Kosmos soll für den Menschen gemacht sein? Würde das nicht heißen, dass Gott ein miserabler Künstler sein muss, wenn er sich derart überdimensionierte Bühne schafft, um ein lausiges Theaterstück, genannt Menschheitsgeschichte, aufzuführen?" Hans-Dieter Mutschler, Halbierte Wirklichkeit. Warum der Materialismus die Welt nicht erklärt? (Kevealer: Butzon \& Bercker, 2014), $312 \mathrm{f}$.

${ }^{5}$ Wolfhart Pannenberg, Was ist der Mensch? Die Anthropologie der Gegenwart im Lichte der Theologie (Göttingen: Vanderhoeck \& Ruprecht, ${ }^{7}$ 1985), 21 f.

${ }^{6}$ Balázs Mezei, Mai vallásfilozófia [Heutige Religionsphilosophie] (Budapest: Kairosz, 2010), 144 ff., mit Anm. 83.

${ }^{7}$ John Lennox, Hat die Wissenschaft Gott begraben? Eine kritische Analyse moderner Denkvoraussetzungen (Deutsch von Jan Carsten Schnurr). (Wuppertal: R. Brockhaus Verlag, $\left.{ }^{4} 2004\right), 36 \mathrm{ff}$. „Die Wörter »nichts als«, »einzig« und »einfach« sind die heimliche Unterschrift eines ontologischreduktionistischen Denkens. Wenn wir diese Wörter entfernen, dann bleibt uns gewöhnlich eine Aussage, gegen die nichts einzuwenden ist. Das Universum ist sicherlich eine Ansammlung von 
sich zuerst überschreiten, überbieten, um dann zwei Schritte zurücktreten zu können. Welches andere Lebewesen ist imstande, seine spezielle Lage auf Erden und im Universum nicht einfach zu behaupten, sondern gerade zu leugnen? Man muss im Zusammenhang mit der Religion auch vom Ursprung der Sprache sprechen. Jede Form von Religion setzt ein gewisses Niveau des begrifflichen Denkens voraus. ${ }^{8}$ Die Grundfragen aller denkenden Menschen sind: woher, wohin, warum/wozu? Warum ist es in den meisten Religionen mehr oder weniger gemeinsam, dass der Mensch in der Vergangenheit viel glücklicher war, als er näher zu Gott/zu den Göttern lebte, und dass er zu jenem „goldenen Zeitalter“ wiederfinden muss? Wozu kann das nichts mehr als eine Illusion sein? ${ }^{9}$ Wenn es um die Frage geht: Warum ist der Mensch religiös, muss also die nächste Frage nicht so lauten: Was ist Religion? sondern, nochmals mit Pannenberg: Was ist der Mensch? ${ }^{10}$ Das ist der erste Schritt in die Richtung und in der Hoffnung, ontologisch-reduktionistische Fehlinterpretationen wie die der Neuen Atheisten zu vermeiden. ${ }^{11}$ Fragen wir auf den Spuren von Karl Barth: Was ist wichtiger? Das, was uns mit den Tieren verbindet oder was uns von ihnen unterscheidet bzw. trennt? ${ }^{12}$ Also,

Atomen, und Menschen vermehren in der Tat DNS. Diese beiden Aussagen sind Aussagen der Wissenschaft. Doch sobald wir die Wörter »nichts als« hinzufügen, gehen die Aussagen über die Wissenschaft hinaus und werden Ausdrucksweise eines naturalistischen Glaubens.“ (37.)

${ }^{8}$ Kurz gesagt mit Robert N. McCauley, Why Religion Is Natural and Science Is Not (OxfordNew York: Oxford University Press, 2011), 172.: „That religious systems largely depend on linguistic communication comes as no surprise. So does science. Religion is a human undertaking and human beings are indefatigable language users." - Mit JohnBowker, Why Religions Matter (New York: Cambridge University Press, 2015), 13. denke man an die Sprache im Sinne von Vorstellung, Einbildung, die einen massiven Beitrag zum Überleben der Menschheit leistet. „... it is because religions have created the many different worlds of imagination, practice and belief in which humans have lived.“ Und 281.: „Religions, to repeat the point [...] are the earliest protective systems of which we have evidence that create the circumstances in which people are likely to survive, to have children and to bring them up to be adults."

${ }^{9}$ „Religion ist für viele etwas Schönes, Wertvolles, Notwendiges. Wenn sie aber eine Illusion ist, ist sie doch nur eine Illusion, auch wenn sie noch so schön ist. Die Wahrheitsfrage darf nicht die zweite sein." Dietrich Bonhoeffer, Andacht zum Semesterbeginn, GS 4, 142 f.

${ }^{10}$ S. Anm. 5.

${ }^{11}$ Vgl. Szentpétery, „Weltethos, neuer Atheismus und christliche Identität,“ 76-82.

12 Die Fragestellungen und Überlegungen von Karl Barth (im Blick auf den Siegeszug des Darwinismus im 19. Jahrhundert) sind auch heute angesichts genötigter und übertriebener (Ideologisierungen der) Globalisierungsprozesse aktuell geblieben: „Wir müßten schon vorweg erkannt haben, daß wir Menschen und als Menschen dem Tier und allen anderen Wesen gegenüber etwas ganz Eigenes und ganz Anderes sind, um uns dann in diesem Phänomen des Menschlichen wiederzuerkennen. Symptome einer Sache nimmt nur der wahr, der mit dieser Sache vertraut ist. [...] Das 19. Jahrhundert war darum ein düsteres Jahrhundert ohnegleichen, weil es die Zeit war, in der der Mensch gleichzeitig mit einem ruckartigen Fortschritt in der Entfaltung seiner Mög- 
die Antwort hängt von unserer jeweiligen weltanschaulich-religiösen Einstellung ab. Aber die Wahrheit ist exklusiv. Ein Wahrheitsrelativismus, der für sich sowas wie einen absoluten Wahrheitsanspruch erhebt, hat ,seine Aporetik darin. “" ${ }^{3}$

Das ständig wiederkehrende Problem ist der natürliche vs. übernatürliche Ursprung der Moralität. Es wird immer wieder behauptet, dass ein anständiger, sittlicher Mensch überhaupt nicht religiös zu sein brauche, und dadurch der übernatürliche Ursprung des sittlichen Handels schon widerlegt sei. Aber eine andere nicht weniger logische Erklärungsmöglichkeit sollte nicht von vornherein ausgeschlossen werden: Sittlichkeit sitzt so tief im Inneren des Menschen, dass sie in vielen Fällen auch noch erhalten bleibt, wenn der ursprüngliche Grund für ihren Träger als unnötig erscheint. Und verweise man hier auf das Projekt Weltethos. Die goldene Regel ist in verschiedenen Formen aus vielen Religionen bekannt. Theologisch formuliert: Religionswissenschaft erschließt, wie weit das ins Herz geschriebene Gesetz reicht. ${ }^{14}$ So könnte man das Projekt Weltethos als eine umfassende und tiefgreifende Anwendung der Religionswissenschaft bezeichnen.

\section{THEOLOGIE, RELIGIONSWISSENSCHAFT UND OBJEKTIVITÄT}

Es wurden schon Unmengen von Büchern und Aufsätzen über diese Thematik, vom Unterschied zwischen Theologie und Religionswissenschaft in Gegenstand, Methode und Kompetenz geschrieben. Doch seien hier ein paar Bemerkungen erwähnt, weil jeder Theologe für sich und für seine Studenten diese Frage immer wieder erneut klären muss. In einer kirchlichen Hochschule werden Religionsgeschichte, Religionswissenschaft, Religionsphilosophie freilich im Rahmen der Theologie unterrichtet und nicht umgekehrt. Vor allem ist die Klärung der Rolle bzw. der Funktion der Theologie nötig. Theologie ist die rationale Reflexion der religiösen Wirklichkeit innerhalb

lichkeiten sich selbst ein Unbekannter wurde. [...] Kennt der Mensch sich nicht schon zuvor lange bevor er sich mit jenen Phänomenen [des Menschlichen] beschäftigt, - dann wird er mit sehenden Augen blind sein, er will dann angesichts und trotz dieser Phänomene immer wieder auf die verkehrte Seite fallen, d. h., immer wieder meinen, sich überzeugen zu müssen, seine eigene Wirklichkeit bestehe in dem, was er mit dem Tier, mit dem übrigen Kosmos überhaupt gemeinsam hat." KD III/2 $104 \mathrm{f}$.

${ }^{13}$ Vgl. Werner Thiede, Die Wahrheit ist exklusiv. Streitfragen des interreligiösen Dialogs (Giessen: Brunnen Verlag, 2014), 5.

${ }^{14}$ S. Martin Bauschke, Die Goldene Regel. Staunen - Verstehen - Handeln (Berlin: EB-Verlag, 2010), 31-60. 
der religiösen Wirklichkeit. ${ }^{15}$ Könnte aber die im weitesten und breitesten Sinne verstandene Religionswissenschaft ein überzeugendes, nicht mehr zu hinterfragendes Bild vom Ursprung der Religion liefern, dann wäre sie „die“ Theologie.

Der Gegenstand der Theologie ist selbstverständlich Gott. Er kann aber nicht in die Hand genommen, „obduziert“, zum Gegenstand wiederholbarer Beobachtungen und Experimente gemacht werden. Oder zum Teil doch? Wir können von Gott nur im Verhältnis zum Menschen bzw. zur Welt sprechen, deshalb ist Theologie zugleich Anthropologie. Gotteserkenntnis ist nur in dem Maße möglich, in welchem Gott sich mit dem Menschen bekannt macht. Also, durch die geschaffene Welt, durch sein Wirken in der Geschichte und im Leben des Einzelnen, ab und zu durch das, was man Wunder nennt - aber alles ist „nur“ im Glauben erkennbar und entzieht sich festen Formulierungen. ${ }^{16}$ Nach der elementaren Logik, die manchmal in Vergessenheit zu geraten sein schien: Wenn es ein unendlich intelligenteres Wesen als wir gibt, hängt es ausschließlich von seiner Einsicht ab, wieviel es von sich für uns bekannt zu werden zulässt. Wie man wohl weiß, erstreckt sich das Spektrum der (beschränkten oder beschränkenden) Beurteilung der möglichen Entzifferung der Offenbarung vom Agnostizismus über die Unbeweisbarkeit bis zur Beweisbarkeit der Existenz Gottes mit Hilfe der Naturwissenschaften. ${ }^{17}$

${ }^{15} \mathrm{Vgl}$. Mezei, Mai vallásfilozófia, (s. Anm 6) $34 \mathrm{f}$.

${ }^{16}$ Vgl. z. B. James W. Jones, Can Science Explain Religion? The Cognitive Science Debate (Oxford-New York: Oxford University Press, 2016), 181. Er argumentiert, dass religiöse Erklärungen logisch und berechtigt sind, obwolh es nicht sein Ziel ist, religiöse Behauptungen zu „beweisen“ Davor 177 f.: Was das Verhältnis von (Natur)wissenschaft und Religion angeht: „There is a similarity between science and religion: at a very general level they both help us make sense of our experiences in the world. But the types of experience they each focus on are very different. [...] Religion is not about making sense of laboratory experiences or statistical analyses.“

${ }^{17}$ Nur je ein Beispiel zur Reflexion über die beiden letzteren: „Der heute so genannte Urknall ist ein beliebiger Punkt, auf einer gigantischen Ursache-Wirkungs-Kette, den nichts auszeichnet, als dass unsere kognitiven Kompetenzen zufällig hier ans Ende gekommen sind. Warum sollten wir gerade an diesem Punkt nach Gott suchen, wo die menschliche Intelligenz zu Ende ist und unsere Borniertheit anfängt? Wenn die christliche Schöpfungslehre wahr ist, dann ruft Gott die Welt nicht nur ins Sein, sondern er ist koextensiv mit ihr und trägt sie in jedem Augenblick, an jeder Stelle und zu jeder Zeit. Warum sollte er dann gerade an einer individuellen Raum-ZeitStelle besonders präsent sein, die sich durch nichts anders als unsere Unwissenheit auszeichnet?“ Mutschler, Halbierte Wirklichkeit, (s. Anm 4) 286 f. Werner Gitt stellt umfassend dar, dass es möglich ist, Naturgesetze für nichtmaterielle Größen, also auch für die - strikt definierte Information zu formulieren und sie auch auf die Lebewesen anzuwenden. Information braucht immer einen intelligenten Sender, der diese zu einem von ihm erwünschten Ziel schafft und nutzt. „Für die Information in den Lebewesen ist allein aufgrund von Naturgesetzen ein allwissender, 
Persönlich halte ich es für wichtig, den Studenten immer wieder in Erinnerung zu rufen und in verschiedenen Kontexten zu zitieren, worauf uns Professor Károly Prőhle aufmerksam machte, als Theologie unter der Herrschaft der quasi Staatsreligion des Marxismus-Leninismus nicht als Wissenschaft galt: „Theologie beschäftigt sich mit der Wirklichkeit, sie will die Wirklichkeit ergreifen. " ${ }^{18}$ So einfach ist es. Es geht also nicht darum, dass man in einem gewissen Kreis ein Gesellschaftsspiel spielt. Denn wir würden damit weder Gott noch uns selber ernst nehmen. So banal das klingen mag, muss es doch den künftigen Pfarrern, Religionslehrern und Gemeindemitarbeitern bewusst gemacht werden, um sie zur selbständigen Reflexion, also zur Denkfähigkeit, Urteilsbildung zu veranlassen. Dazu gehört, dass das Streben nach richtig gemeinter Objektivität zur Selbstverständlichkeit werden muss. Ein besonders schönes Beispiel ist dazu was Bonhoeffer mit dichterischer Erhebung zum Hintergrund der indischen Religion sagte: „Die Welt, in der die tätig schaffende Hand der Arbeit sich nur zu öffnen braucht, um die Früchte reichlich zu empfangen, stiftet ihren Bewohnern eine große Stille ins Herz, und die Seele atmet das Leben, das sie in seiner Fülle umgibt, sie dringt ein in dieses volle, gebende Lebensgeschehen, eint sich mit ihm, durchforscht und ergrübelt seine Rhytmen und seine Tiefen, die im Grunde die Tiefen der Seele selbst sind, und die Weiten der indischen Seele sind die Weiten alles Lebendigen. So erkennt sich die sich versenkende Seele wieder in allem Lebendigen wie in tausend Spiegeln, aus jeder Gestalt der Natur vernimmt sie die stille Antwort: tat tvam asi, das bist du, du selbst."19 Also, andere Religionen, religiöse Erscheinungen müssen zuerst unter ihren eigenen Umständen und Zusammenhängen, also angesichts ihrer breitesten Entstehungsgeschichte gedeutet werden. Dazu muss man die Ergebnisse des jeweiligen Standes der Religionswissenschaft zur Hilfe rufen. Verständnis für andere Religionen braucht natürlich nicht unbedingt (oder manchmal darf

\footnotetext{
allmächtiger und ewiger Sender zwingend erforderlich.“ Werner Gitt, Information. Der Schlüssel zum Leben (Bielefeld: Christliche Literatur-Verbreitung, 2016), $294 \mathrm{f}$.

${ }^{18}$ Károly Prőhle d. J. (1911-2005), sein Vater Károly Prőhle d. Ä. (1875-1962) war Professor für Systematik an der Evangelisch-lutherischen Hochschule, dann Fakultät in Sopron (Ödenburg), Westungarn. Nach der Absolvierung studierte er in Tübingen, Königsberg, Halle und Erlangen, war Pfarrer in mehreren Gemeinden, ab 1950 an dem Rechtsnachfolger der Fakultät, der EvangelischLutherischen Akademie in Budapest. Zuerst war er Professor für Praktische Theologie, dann am längsten für Neues Testament, schliesslich für Systematik, ausserdem unterrichtete er Philosophie und Religionsgeschichte bis 1998. 1972-1982 war er Generalsekretär des Ökumenischen Rates der Kirchen in Ungarn, und nahm u. a. an der Ausarbeitung des Lima-Dokuments teil.

${ }^{19}$ Dietrich Bonhoeffer, Das Recht auf Selbstbehauptung, GS 3,261 ff. Vgl. Szentpétery, „Dietrich Bonhoeffer und der heutige religiöse Pluralismus,“ $126 \mathrm{f}$.
} 
ausdrücklich nicht) mit Einverständnis gleichgemacht zu werden. Man hat Angst vor allem vor dem Unbekannten, aber ab und zu doch etwas mehr vor dem Bekannten, denkt man an religiöse Extremisten oder extreme neuere religiöse Erscheinungen bzw. Psychokulte.

Eine hundertprozentig vollkommene Objektivität, Neutralität ist in der Beschäftigung mit Religionen nicht möglich, auch nicht einmal mit der Absicht eines nur beschreibenden Charakters, wie auch keine hundertprozentige Sicherheit. ${ }^{20}$ Deshalb müsste man sich - wie ich einmal von einem Sicherheitsexperten hörte - nach einer hundertzwanzigprozentigen streben. Ein offensichtliches, extremes Beispiel für die nie perfekte Objektivität ist die Beschäftigung mit der Praxis des Menschenopfers. Ein christlicher Theologe überhaupt oder ein Religionswissenschaftler als Bürger eines demokratischen Rechtstaates kann so etwas heute nicht akzeptieren, ohne das in aller Offenheit zum Ausdruck bringen zu müssen, das kommt einfach nicht in Frage. ${ }^{21}$ Neutralität ist nicht gleich mit Agnostizismus und bei weitem nicht mit Atheismus.

${ }^{20}$ Was die Entstehungsgeschichte unserer „Religion“ angeht, kann man jederzeit mit Karl Barth über die Auferweckung Jesu fragen: „Gibt es so etwas wie eine »historische«, d. h. neutral und objektiv feststellende Erkenntnis dieses Ereignisses? Gewiß nicht [...] als echte und fruchtbare Erkenntnis der Liebe zu dem in ihm sich offenbarenden Gott. [...] Liebe erkennt nicht neutral, nicht objektiv. Ein neutrales, objektives, ein »historisches« Erkennen ist aber ihre Voraussetzung." Dazu muss man die neutestamentlichen Texte möglichst unbefangen und gewissenhaft erforschen. Unbefangenheit heisst $\mathrm{u}$. a., dass man den Texten sagen lässt, was sie sagen wollen, und nicht seine Vorentscheidungen in sie hineinliest. ,»Historische « Erkenntnis in dieser Unbefangenheit wird, weil zunächst niemand unbefangen ist, nie eine leichte und selbstverständliche Sache sein. Man wird aber nicht sagen dürfen, daß die Erfüllung dieser Bedingung zum vornherein unmöglich ist." Vgl. KD IV/2 167. Man könnte zugespitzt formulieren, dass Befangenheit, Voreingenommenheit in welche Richtung auch immer höchstens einundfünfzig Prozent erreichen darf. - Edmund Schlink, Ökumenische Dogmatik (Göttingen, Vanderhoeck \& Ruprecht $\left.{ }^{2} 1985\right)$, 364. setzt sich für einen anderen Ausgangspunkt ein. Er macht u. a. darauf aufmerksam, dass die Prinzipien der Analogie und der durchgängigen Wechselwirkung weder historisch noch philosophisch zu beweisen sind. Sie sind wichtige und nützliche, aber doch nur heuristische, also keine letzgültigen Prinzipien. „Der Beweis, daß die Auferstehung eines Toten unmöglich ist, ist unmöglich. [...] Es kann wissenschaftstheoretisch nicht ausgeschlossen werden, bei der Erforschung der neutestamentlichen Erscheinungsberichte den Einsatz bei der Gestalt des Erschienenen zu nehmen, und von diesem Vorverständnis her die Methoden historischer Forschung anzuwenden.“

${ }^{21}$ Vgl. Péter Szentpétery, "Theology of Religions, Religious Studies and Dialogue of Religions“ w Theories and Trends in Religions and in the Study of Religion, (ed.) Bulcsú K. Hoppál (Budapest: L'Harmattan, 2015), 232. 


\section{DIALOG MIT ANDEREN RELIGIONEN UND IN DER ÖKUMENE}

Die bisher Gesagten führen in den Bereich des interreligiösen Dialogs, bei dem man sie vor Augen halten muss. Religion existiert freilich nicht als eine abstrakte Größe, sondern es gibt Menschen verschiedener Religionen, die auch über ihre eigene Religion nur mehr oder weniger im Klaren sind. So, es ist nicht immer einfach, den entsprechenden Gesprächspartner zu finden, man kann und muss mit denjenigen ins Gespräch kommen, dem man begegnet. Doch können persönliche Begegnungen, Bekanntschaften nicht einmal durch die beste, gründlichste und zeitgemäßeste Fachliteratur ersetzt werden. ${ }^{22}$ Verständnis und Versöhnung ist nur mit gegenseitigem Ernstnehmen möglich, man muss aber dazu zuerst seine eigene Überzeugung ernst nehmen. Echter Dialog ist nicht gleich mit Selbstaufgabe, Synkretismus und am Ende mit einem Wirrwarr im Kopf und Herzen. Wenn jemand einzelne Religionen oder überhaupt Religiosität, religiöse Phänomene studiert, führt man schon auch wenn unbewusst, Dialog mit ihnen, mit dem Buch in der Hand oder mit dem Bildschirm vor den Augen. Und die von Vertretern anderer Religionen - entweder aus mangelnder Kenntnis oder aus verständlichen Gründen - gestellten Fragen und sogar falsche Behauptungen helfen sehr viel, sich im eigenen Glauben zu vertiefen.

Dazu ein Paar Worte zur Toleranz mit Theo Sundermeier. Toleranz ist keine in allen Orten und Zeiten in gleicher Weise verstandene Kategorie. Er teilt sie in drei Aspekte: 1. Der Einzelne besitzt die gleiche Würde wie die Gruppe selbst. 2. Religion ist eine Angelegenheit des Einzelnen und jeder ist seinem Gewissen verantwortlich. 3. Die Glaubensüberzeugung hat etwas mit Wahrheit zu tun, die zu anderen Wahrheiten in Konkurrenz steht und von ethischen und rituellen und $d$. h. vom Sozialverhalten getrennt werden

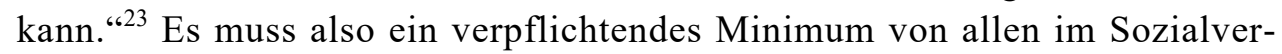
kehr anerkannt werden.

Mutatis mutandis gelten die Richtlinien der Begegnung mit Vertretern anderer Religionen auch für den ökumenischen Dialog. Mögliche Objektivität und Profilierung, Ökumene der Profile ergänzen sich einander auf diesem Wege. Es muss erwähnt werden, dass es in den Prinzipien keine scharfe Grenze

\footnotetext{
${ }^{22}$ Vgl. Szentpétery, „Weltethos, neuer Atheismus und christliche Identität,“ 75. zu Robert Spaemann, „Weltethos als »Projekt«"“ Merkur. Deutsche Zeitschrift für europäisches Denken 50(1996), 9-10: 898. Um sich über das Verbindende klarzuwerden, meint er, bedürfe es weder ausgedehntes Nachdenkens, noch institutionalisierter Dialoge.

${ }^{23}$ Theo Sundermeier, Was ist Religion? Religionswissenschaft im theologischen Kontext. Ein Studienbuch (Gütersloh: Chr. Kaiser, Gütersloher Verlagshaus, 1999), 192.
} 
zwischen ökumenischem und interreligiösem Dialog gibt, doch ist es wichtig, dass die Gesprächspartner im Ökumenischen durch viel mehr verbunden als getrennt sind. Interreligiöser Dialog ist sowieso längst Teil der Ökumenischen Bewegung geworden. Kein protestantischer Theologe kann z. B. die Erklärung Nostra aetate des Zweiten Vaticanum (1965) als Basistext für den Dialog der römisch-katholischen Kirche mit den nichtchristlichen Religionen außer Acht lassen. Ähnliches gilt für die Dominus Iesus Erklärung der Glaubenskongregation, (2000), obwohl sie in letzterer Zeit nur selten erwähnt wird, wo ausgegangen aus der Einzigartigkeit Christi und der Ablehnung einer pluralistischen Theologie der Religionen dann im Kapitel IV über die Mangelhaftigkeit, defectus aller anderen Kirchen die Rede ist. ${ }^{24}$ Im interreligiösen Dialog muss man die (m. E.) Schlüsselsätze der beiden (dialektisch) vor Augen halten: 2. „Die katholische Kirche lehnt nichts von alledem ab, was in diesen Religionen wahr und heilig ist. Mit aufrichtigem Ernst betrachtet sie jene Handlungs- und Lebensweisen, jene Vorschriften und Lehren, die zwar in manchem von dem abweichen, was sie selber für wahr hält und lehrt, doch nicht selten einen Strahl jener Wahrheit erkennen lassen, die alle Menschen erleuchtet.“25 22. „Die Parität, die Voraussetzung für den Dialog ist, bezieht sich auf die gleiche personale Würde der Partner, nicht auf die Lehrinhalte und noch weniger auf Jesus Christus, den menschgewordenen Sohn Gottes, im Vergleich

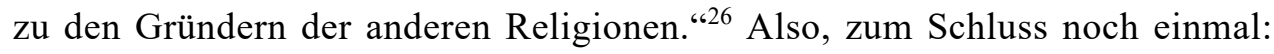
maximales Verständnis aber keine Selbstaufgabe.

\section{ZUSAMMENFASSUNG BZW. THESEN}

1. Jeder Erklärungsversuch des Ursprungs der Religion setzt eine religiösweltanschauliche Grundlegung voraus. Alle haben einen gewissen Wahrheitsgehalt, doch reicht auch ihre Summe nicht aus.

${ }^{24}$ Ulrich Körtner, Ökumenische Kirchenkunde. Lehrwerk Evangelische Theologie 9 (Leipzig: Evangelische Verlangsanstalt, 2018), 324. In der Einleitung erwähnt er, dass „Das etwa von Hans Küng oder dem Herausgeberkreis der Zeitschrift »Dialog der Religion« (1992 ff.) verfolgte Projekt einer Ökumene der Religionen überspielt die konfessionelle Problematik des Christentums noch stärker als manche Entwürfe einer Theorie der sichtbaren Einheit der Kirche.“ (15.)

${ }^{25}$ „Erklärung Nostra aetate über das Verhältnis der Kirche zu den nichtchristlichen Religionen“ [07.02.2021], http://www.vatican.va/archive/hist_councils/ii_vatican_council/documents/vat-ii_ decl_19651028_nostra-aetate_ge.html

$\overline{26}$ „Kongregation für die Glaubenslehre: Erklärung »Dominus Iesus« über die Einzigkeit und Heilsuniversalität Jesu Christi und der Kirche“ [07.02.2021], http://www.vatican.va/roman_curia/ congregations/cfaith/documents/rc_con_cfaith_doc_20000806_dominus-iesus_ge.html 
2. Religion strebt sich nach dem letztgültigen Verständnis des Sinnes der Dinge. Das Menschsein hat nur Sinn wenn auch dieser gewaltige Kosmos Sinn hat.

3. Zur Klärung der Frage: „Warum is der Mensch religiös?“ muss zunächst die Frage: „Was ist der Mensch?“ gestellt werden.

4. Ontologischer Reduktionismus wie etwa „Das Leben ist nichts anderes als“ oder „der Mensch ist nichts mehr als“ ist Selbstwiderpruch. Man muss zuerst eine Perspektive haben, sich überschreiten, um seine Mangelhaftigkeit feststellen zu können.

5. Das bisher Gesagte bezieht sich auch auf den Ursprung der Moraliät des Menschen. Der übernatürliche Ursprung kann nicht durch die Leugnung des Übernatürlichen widerlegt werden.

6. Religionswissenschaft (und Religionsphilosophie) gehört zum theologischen Curriculum. Sie kann dabei helfen, die Natur, Möglichkeit und Grenzen einer objektiven Einstellung in der Forschung der nichtchristlichen Religionen zu verstehen und praktizieren.

7. Religionen müssen unter den Umständen ihres Ursprungs, in ihrer Entstehungsgeschichte verstanden werden. Verständnis kann und darf nicht (immer) mit Einverständnis gleichgesetzt werden.

8. Echter Dialog kann keine Selbstaufgabe und Toleranz keine kritiklose Anpassung bedeuten. Fragen und Kritiken der jeweiligen Gesprächspartner, auch wenn sie nicht berechtigt sind, können zu unserem Selbstverständnis einen wichtigen Beitrag leisten.

9. Ökumenischer und interreligiöser Dialog bedingen einander wechselseitig. Sie werden aufgrund derselben Prinzipien geführt, doch in der Ökumene ist es offensichtlich, dass die Partner - in ihrem Glauben an Jesus Christus, dem menschgewordenen Sohn Gottes - durch viel mehr verbunden als getrennt sind.

BIBLIOGRAPHIE

Barth, Karl. Kirchliche Dogmatik. III/2. Die Lehre von der Schöpfung. Zollikon-Zürich: Evangelischer Verlag, 1948.

Barth, Karl. Kirchliche Dogmatik. IV/2. Die Lehre von der Versöhnung. Zollikon-Zürich: Evangelischer Verlag, 1955.

Bauschke, Martin. Die Goldene Regel. Staunen - Verstehen - Handeln. Berlin: EB-Verlag, 2010.

Bonhoeffer, Dietrich. Andacht zum Semesterbeginn. Gesammelte Schriften. Band 4. Auslegungen Predigten (Hrsg. von Eberhard Bethge), 142-143. München: Christian Kaiser Verlag, 1961.

Bonhoeffer, Dietrich. Das Recht auf Selbstbehauptung. Gesammelte Schriften. Band 3. Theologie Gemeinde (Hrsg. von Eberhard Bethge), 258-269. München: Christian Kaiser Verlag, 1960. 
Bowker, John. Why Religions Matter. Cambridge-New York: Cambridge University Press, 2015.

Erklärung Nostra aetate über das Verhältnis der Kirche zu den nichtchristlichen Religionen. [07.02.2021]. http://www.vatican.va/archive/hist_councils/ii_vatican_council/documents/vat-ii decl_19651028_nostra-aetate_ge.html

Frankl, Viktor E. Der Mensch auf der Suche nach Sinn. Zur Rehumanisierung der Psychotherapie. Freiburg-Basel-Wien: Herder Verlag, ${ }^{4} 1975$.

Gitt, Werner. Information. Der Schlüssel zum Leben. Bielefeld: Christliche Literatur-Verbreitung, 2016.

Jones, James W. Can Science Explain Religion? The Cognitive Science Debate. Oxford-New York: Oxford University Press, 2016.

Kärkkäinen, Veli-Matti. „Meaningful life in a meaningless cosmos? A short reflection for good times - and bad.“ Dialog. Journal of Theology 59 (2020): 103-106.

Kongregation für die Glaubenslehre: Erklärung „Dominus Iesus“ über die Einzigkeit und Heilsuniversalität Jesu Christi und der Kirche. [07.02.2021]. http://www.vatican.va/roman curia/congregations/cfaith/documents/rc_con_cfaith_doc_20000806_dominus-iesus_ge.html

Körtner, Ulrich. Ökumenische Kirchenkunde. Lehrwerk Evangelische Theologie 9. Leipzig: Evangelische Verlangsanstalt, 2018.

Lennox, John. Hat die Wissenschaft Gott begraben? Eine kritische Analyse moderner Denkvoraussetzungen. (Deutsch von Jan Carsten Schnurr). Wuppertal: R. Brockhaus Verlag, 2004.

McCauley, Robert N. Why Religion Is Natural and Science Is Not. Oxford-New York: Oxford University Press, 2011.

Mezei, Balázs. Mai vallásfilozófia. Budapest: Kairosz, 2010.

Mutschler, Hans-Dieter. Halbierte Wirklichkeit. Warum der Materialismus die Welt nicht erklärt? Kevealer: Butzon \& Bercker, 2014

Pannenberg, Wolfhart. Was ist der Mensch? Die Anthropologie der Gegenwart im Lichte der Theologie. Göttingen: Vanderhoeck \& Ruprecht, ${ }^{7} 1985$.

Schlink, Edmund. Ökumenische Dogmatik. Göttingen, Vanderhoeck \& Ruprecht, ${ }^{2} 1985$.

Spaemann, Robert. „Weltethos als »Projekt«.“ Merkur. Deutsche Zeitschrift für europäisches Denken 50(1996), 9-10: 893-904.

Sundermeier, Theo. Was ist Religion? Religionswissenschaft im theologischen Kontext. Ein Studienbuch. Gütersloh: Chr. Kaiser, Gütersloher Verlagshaus, 1999.

Szentpétery, Péter. „Dietrich Bonhoeffer und der heutige religiöse Pluralismus. Roczniki Teologiczne 62(2015), 7: 121-135.

Szentpétery, Péter. "Theology of Religions, Religious Studies and Dialogue of Religions." W Theories and Trends in Religions and in the Study of Religion, ed. Hoppál, Bulcsú K. 229233. Budapest: L'Harmattan, 2015.

Szentpétery, Péter. „Weltethos, neuer Atheismus und christliche Identität.“ Roczniki Teologiczne 66(2019), 7: 69-86.

Thiede, Werner. Die Wahrheit ist exklusiv. Streitfragen des interreligiösen Dialogs. Giessen: Brunnen Verlag, 2014. 


\section{TEOLOGIA, RELIGIOZNAWSTWO I KSZTAŁCENIE TEOLOGICZNE. KILKA MYŚLI, KTÓRE POWINNO SIĘ ODKRYWAĆ NA NOWO}

$$
\text { Streszczenie }
$$

W artykule zebrano kilka refleksji, uznanych przez autora za ważne w kontekście religijności i religii w kształceniu teologicznym. Z pierwszej części płynie wniosek, że przyszli księża, osoby pracujące w Kościele i nauczyciele religii muszą zdawać sobie sprawę, że zawsze będą stykać się z pytaniami o źródła religijności, człowieczeństwa i moralności. Kluczową kwestią jest pytanie kim jest istota ludzka. W drugiej części autor udziela odpowiedzi na pytanie o znaczenie obiektywizmu dla religioznawstwa i czym się on różni w teologii. Nie można go utożsamiać z agnostycyzmem czy nawet ateizmem. Każdy, kto studiuje teologię, musi zapoznać się z innymi religiami, uwzględniając ich kontekst i źródła, wykazując maksimum zrozumienia i empatii, które nie muszą oznaczać zgody. Takie racje prowadzą nas do trzeciej części artykułu, gdzie mowa o dialogu międzywyznaniowym i ekumenicznym. Kontaktu osobistego nie zastąpi nawet najbardziej aktualna literatura. Dialog nie oznacza rezygnacji z samego siebie, bo dialog wtedy nie byłby uczciwy. Zasady rządzące dialogiem ekumenicznym (tzn. międzywyznaniowym) są zasadniczo takie same, choć zdecydowanie bardziej łączą niż dzielą uczestników dialogu. Krótko mówiąc - jesteśmy poszukiwaczami prawdy. W podsumowaniu autor zawarł dziewięć stwierdzeń.

Słowa kluczowe: teologia; religioznawstwo; religijność; źródła religii; dialog; moralność; obiektywizm; tolerancja. 